\title{
EU:s sammanhållningspolitik och regionala skillnader 2006-2016
}

\author{
DANIEL RAUHUT ${ }^{1} \&$ NUNO COSTA ${ }^{2}$ \\ Itä-Suomen yliopisto ${ }^{1}$, Universidade de Lisboa ${ }^{2}$
}

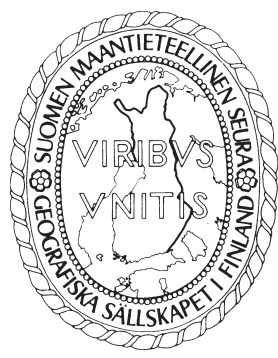

Rauhut, Daniel \& Costa, Nuno (2020). EU:s sammanhållningspolitik och regionala skillnader 2006-2016 (The EU cohesion policy and regional differences 2006-2016). Terra 132: 4, 175-189. https://doi.org/10.30677/terra.89774

$\mathbf{y}$ The effects of the EU Cohesion Policy are debated, and while some studies find positive effects, others point to negative effects. Many of the methodological approaches that have so far been used to measure the EU Cohesion Policy are simple benchmarking exercises, focusing on a few indicators covering a short time period. Other evaluations are fragmented, qualitative in nature, and narrow in scope. This paper aims at analysing the EU Cohesion Policy and its impact for the period 2006-2016. We construct a Territorial Cohesion Development Index with target posts in order to analyse the impact at a NUTS2 level. The findings indicate that bigger cities and highly urbanised regions in Central Europe benefited the most from the policy, while non-core and peripheral regions fell further behind.

Key words: cohesion policy, Territorial Cohesion Development Index, core-periphery, regional development

Daniel Rauhut, Karelian Institute, University of Eastern Finland, P.O. Box 111, FI-80101 Joensuu, Finland. E-mail: <daniel.rauhut@uef.fi>

När den europeiska sammanhållningspolitiken (ECP) introducerades 1989 var den utformad för att stimulera ekonomisk och social sammanhållning (CEC 2014). Behovet av att sammanhållningspolitiken även skulle omfatta en territoriell eller rumslig dimension framträdde i samband med att "European Spatial Development Perspective" (ESDP) skapades (Faludi 2006). Ett formellt erkännande av den territoriella dimensionens betydelse för den europeiska sammanhållningspolitiken kom i samband med Lissabonfördraget 2009 då "territoriell sammanhållning" lades till de båda policymålen om ekonomisk och social sammanhållning (Mendez 2011; Faludi 2016a).

Effekterna av ECP är omdiskuterade. Flera studier har pekat på positiva effekter. Detta gäller särskilt för perioden 2000-2006 för regioner nära urbana agglomerationer (Gagliardi \& Perocco 2017). Även om effekterna tycks vara små, så varar effekterna från de av Strukturfonderna finansierade insatserna länge (Bradley 2006), och den allmänna uppfattningen är att ECP haft en positiv inverkan på utveckling och ekonomisk tillväxt (Becker m.fl. 2012; Pellegrini m.fl. 2012; Ward \& Wolleb 2010; Molle 2007; Bachtler m.fl. 2009; Cappelen m.fl. 2003). Insatserna enligt Mål 1 tycks ha haft en positiv inverkan på ekonomisk tillväxt, medan de sammantagna insatserna enligt Mål 1, 2 och 3 inte visar på någon effekt (Mohl \& Hagen 2010).

Flera utvärderingar har pekat på effektivitetsproblem hos ECP. I en jämförande analys av 15 regioner över fyra programperioder (1989-2012) identifierar Becker m.fl. (2012) en brist på konceptuellt tänkande och strategisk motivering för programmen. Syften specificeras inte och inte heller formuleras några mätbara mål. Vidare identifieras institutionella begränsningar för implementeringen av ECP och brister i styrning inom de flesta områden. Vidare har andra studier ifrågasatt effektiviteten i ECP och då särskilt när det gäller politikens 
förmåga att stimulera regional utveckling och en konvergens mellan de europeiska regionerna på lång sikt (Bakucs m.fl. 2018; Becker m.fl. 2018).

I andra utvärderingar dras slutsatsen att de rikaste regionerna har tjänat mera på ECP och dess stödformer än de fattiga (Medve-Bálint 2016), trots att merparten av EU-stöd går till svaga och stagnerade regioner. Dessutom innebär den platsbaserade strategin som antagits efter Barca-rapporten (Barca 2009) att lokala aktörer ges ett ökat ansvar för den territorialiserade ekonomiska och sociala agendan. Detta gör att utmaningarna i perifera regioner blir ett ansvar för de lokala myndigheterna. Tyvärr har dessa aktörer, vanligtvis kommuner, inte verktyg för att möta dessa utmaningar (Solly 2016; Gruber m.fl. 2018; Isola m.fl. 2017). I perifera regioner är det oftast regionhuvudstaden som gynnas av EUstöden, medan EU-stöden inte når regionhuvudstadens omland (Nagy \& Benedek 2021).

Dessvärre behandlar flertalet studier angående effektiviteten inom sammanhållningspolitiken endast kortare tidsperioder, är kvalitativa i sin utformning och fokuserar på ett fåtal indikatorer. Enkla benchmarkingpresentationer för olika indikatorer presenteras i flertalet sammanhållningsrapporter (CEC 2001, 2004, 2007, 2010b, 2014, 2017). I den benchmarkingstudie Dijkstra och Athanasoglou (2015) genomför studeras hur väl EU:s medlemsländer lyckats uppnå Europa 2020-målen. Endast fem indikatorer analyseras: sysselsättning, utbildning, fattigdom, innovationer, samt klimatförändringar och hăllbar energi. Om medlemslandet uppnått målen ges värdet 100, medan ett resultatförverkligande långt ifrån de uppsatta målen ges värdet noll.

Den internationella finanskrisen 2009 visade att krisers effekter inte fördelas jämnt sett ur ett rumsligt perspektiv. Vissa länder drabbades hårdare av krisen än andra, och på en regional nivå drabbades även där vissa regioner hårdare än andra (Hadijmichalis 2011). De positiva effekterna som ECP visat för åren före krisen blev svagare under krisen, och konvergensmålen blev svårare att upprätthålla (Becker m.fl. 2018). Regioner med ekonomiskt diversifierade städer visade på en större uthållighet gentemot den ekonomiska och finansiella krisen 2009 än regioner med endast mindre städer och städer utan diversifierad ekonomisk struktur $(\mathrm{Ca}-$ pello m.fl. 2015). Nedgången för de stora städerna i Europa efter krisen 2009 hör samman med en minskad avkastning relativt städernas storlek, och att de städer som klassificeras som "secondrank cities" kunnat åtnjuta en ökande avkastning relativt sin storlek (Dijkstra m.fl. 2013). Det finns empiriska belägg för att större städer, vanligtvis huvudstaden, spelar en betydande roll för den nationella ekonomiska utvecklingen oavsett vilken period som studeras, men "second-rank cities" spelar en betydande roll under vissa avgränsade perioder (Camagni \& Capello 2015b).

Efter den finansiella krisen 2009 förändrades fokus för ECP, från sammanhållning till konkurrens och från svagare regioner till städer. Följden har blivit en politik som fokuserar på städer och stadsregioner (Faludi m.fl. 2015). Empiriska studier visar att ju mer polycentriskt ett land är, desto större är de regionala skillnaderna inom ett land (Meijers och Sandberg 2008). Värt att notera är att få-EU länder har ett tydligt polycentriskt stadssystem, vilket medför att politiken för att stimulera regional utveckling stöter på implementeringsproblem i monocentriska länder utanför Centraleuropa.

Vid upprepade tillfällen har behovet av ett mått eller metod för att mäta konsekvenserna av ECP rests (t.ex. Faludi 2016b; Hanell 2015; Hamez 2005). De få försöken att mäta konsekvenserna av ECP har antingen utgjorts av deskriptiva benchmarkingmetoder, med hjälp av spindeldiagram (Zaucha \& Böhme 2020) eller kännetecknats av en oklar metodologi (Medeiros 2013). Dock kan dessa vara nyttiga för att utveckla en förståelse för ett problem, men som analytiskt redskap är de otillräckliga. Vad som behövs är ett kvantitativt mått eller metod, som inbegriper ECP:s nyckelindikatorer. Syftet med denna studie är därför att analysera den europeiska sammanhållningspolitiken och dess konsekvenser på regional nivå (NUTS2) för perioden 2006-2016, det vill säga före och efter den finansiella krisen 2009. Följande frågor kommer att besvaras: (1) Vilken typ av regioner vann på den europeiska sammanhållningspolitiken under den analyserade perioden? (2) Vilken typ av regioner förlorade på den förda politiken under samma tidsperiod? (3) Vilka är de troliga orsakerna till att olika slags regioner vann respektive förlorade på den europeiska sammanhållningspolitiken?

Ett index, Territorial Cohesion Development Index (TCDI), kommer att konstrueras för att besvara frågorna. I sin tekniska konstruktion bygger TCDI på samma metodologi som FN:s Human Development Index (HDI). HDI utvecklades för att fokusera på människor och deras möjligheter i livet. För att kunna bedöma människors möjligheter att utveckla sig och uppnå något i livet måste man se bortom BNP. På det sätt HDI är konstruerat kan det även användas för att ifrågasätta nationella policys genom att jämföra två länder på samma ekonomiska utvecklingsnivå men med helt olika resultat i HDI (UNDP 2016a). Mot denna bakgrund kan TCDI användas för att analysera vissa policyval som ECP stipulerar, samt hur regioner på samma ekonomiska nivå kan uppvisa olika resultat sett ur ett sammanhållningspolitiskt perspektiv. 


\section{Indikatorer för att uppnå 'sammanhållning'}

Inom flera områden finns explicit formulerade mål och indikatorer för hur 'sammanhållning' ska uppnås inom EU. Det gäller bland annat sysselsättning, arbetslöshet, utbildning, fattigdom samt klimatförändring och hållbar energi (CEC 2010a). Vidare finns policymål för andelen hushåll inom EU med tillgång till bredband (CEC 2017). Dessa sex indikatorer är därmed relevanta för denna studie. Det är dock svårt, vilket Dijksta och Athanasoglou (2015) påtalat, att analysera klimatförändring från ett sammanhållningsperspektiv.

Sex av de indikatorer som kommer att användas i denna studie hämtas från policydokument i vilka EU-kommissionen har formulerat mål för sammanhållningspolitiken samt identifierat indikatorer för att mäta måluppfyllelsen i den förda politiken: sysselsättningsgraden ska vara minst 75 procent, minst 40 procent av befolkningen mellan 30 och 34 år ska ha eftergymnasial utbildning, andelen av befolkningen som riskerar fattigdom och social exkludering får inte överstiga 19,5 procent (CEC 2010a), ungdomsarbetslösheten ska hållas under 10 procent (CEC 2016) och minst 20 procent av den använda energin ska vara förnyelsebar (CEC 2010a). Vidare ska minst 50 procent av hushållen ha tillgång till bredband (CEC 2017). Dessa indikatorer kan även användas för att mäta vilka konsekvenser ECP haft på regional nivå.

Eftersom alla potentiellt möjliga indikatorer för att mäta vilka konsekvenser sammanhållningspolitiken haft inte explicit mäter 'sammanhållning' har fem "territoriella nycklar" föreslagits för att stärka den territoriella dimensionen av Europa 2020-agendan och ECP. Dessa nycklar avser (1) tillgänglighet (accessibility), (2) tjänster av allmänt ekonomiskt intresse (services of general economic interest), (3) territoriella förutsättningar (territorial capacities / endowments / assets), (4) city networking, och (5) funktionella regioner.

Tabell 1. Indikatorer med policymål och maximivärden.

Table 1. Indicators with goal posts and max values.

\begin{tabular}{|c|c|c|c|}
\hline Dimension & $\begin{array}{l}\text { Indikator / } \\
\text { Indicator }\end{array}$ & Minimum & Maximum \\
\hline \multirow{3}{*}{$\begin{array}{l}\text { Ekonomisk } \\
\text { Economic }\end{array}$} & $\begin{array}{l}\text { Regional BNP per invånare i köpkraftspariteter (PPS) } \\
\text { Regional GDP per capita in purchasing power parities }\end{array}$ & 0 & 100 \\
\hline & $\begin{array}{l}\text { Sysselsättningsgrad för gruppen } 20-64 \text { år (EMP) } \\
\text { Employment rate age group 20-64 years }\end{array}$ & $>75$ & 100 \\
\hline & $\begin{array}{l}\text { Andel av befolkningen } 30-34 \text { år med eftergymnasial utbildning (EDU) } \\
\text { Share of population with tertiary education }\end{array}$ & $>40$ & 100 \\
\hline \multirow{3}{*}{$\begin{array}{l}\text { Social } \\
\text { Social }\end{array}$} & $\begin{array}{l}\text { Andel av befolkningen } 65+\text { år (AGE) } \\
\text { Share of population } 65+\text { years }\end{array}$ & 0 & 100 \\
\hline & $\begin{array}{l}\text { Andel av befolknings som riskerar fattigdom eller social exklusion (PEX) } \\
\text { Share of population at risk of poverty and social exclusion }\end{array}$ & $<19.5$ & 80 \\
\hline & $\begin{array}{l}\text { Andel arbetslösa } 15-24 \text { år (YOU) } \\
\text { Youth unemployment rate }\end{array}$ & $<10$ & 100 \\
\hline \multirow{3}{*}{$\begin{array}{l}\text { Territoriell } \\
\text { Territorial }\end{array}$} & $\begin{array}{l}\text { Km motorväg per } 1000 \mathrm{~km}^{2}(\mathrm{ROA}) \\
\text { Km motorway per } 1,000 \mathrm{~km}^{2}\end{array}$ & 0 & 100 \\
\hline & $\begin{array}{l}\text { Andel av befolkningen med bredband hemma (INT) } \\
\text { Share of population with access to broadband at home }\end{array}$ & $>50$ & 100 \\
\hline & $\begin{array}{l}\text { Antal läkare per } 100000 \text { invånare (MED) } \\
\text { Number of medical doctors per 100,000 inhabitants }\end{array}$ & 0 & 100 \\
\hline $\begin{array}{l}\text { Hållbarhet } \\
\text { Sustainability }\end{array}$ & $\begin{array}{l}\text { Andel förnyelsebar energi (ENE) } \\
\text { Share of renewable energy }\end{array}$ & $>20$ & 100 \\
\hline
\end{tabular}


Transporter, tillgänglighet, välfärdstjänster och internetlösningar kan härledas från dessa fem "territoriella nycklar" (Böhme et al. 2011; Zaucha et al. 2014). Fyra av de indikatorer som används i denna studie kan härledas från dessa "territoriella nycklar": antalet läkare per 100000 invånare (välfärdstjänster), km motorväg per $1000 \mathrm{~km} 2$ (tillgänglighet, transport, city networking), samt andelen i befolkningen över 65 år (territoriella förutsättningar, välfärdstjänster). Slutligen, en möjlig indikator för att identifiera en regions ekonomiska kapacitet är regional BNP per capita mätt i köpkraftspariteter en "territoriell nyckel" för territoriella förutsättningar.

Samtliga 10 indikatorer är relevanta för denna studie och de kan belysa olika delar av vilka konsekvenser EU:s sammanhållningspolitik har haft på regional nivå. De valda indikatorerna belyser även de tre dimensionerna av ECP: ekonomisk, social och territoriell sammanhållning. Vid sidan av dessa tre dimensioner belyses även hållbarhetsperspektivet $\mathrm{i}$ och med att indikatorn för förnyelsebar energi inkluderas i analysen (se Tabell 1).

\section{Data och reliabilitet}

Ett robust index med hög reliabilitet bygger inte endast på hur indexet konstruerats metodologiskt, utan även på kvaliteten hos den använda statistiken. Två aspekter kan nämnas i detta sammanhang: (1) för att kunna mäta effekter och resultat krävs indikatorer för input och kontext. De valda indikatorerna måste fånga detta för att kunna ingå i ett index som mäter konsekvenser (Marques da Costa m.fl. 2013). (2) Inget index är bättre än kvaliteten på den statistik som används. Tillgängligheten på relevant statistik till denna studie är anmärkningsvärt god. Denna studie använder samma statistik på NUTS2-nivån som de ekonometriska studierna av ECP:s effektivitet och resultat som diskuterades i litteraturöversikten ovan. Statistik på NUTS3-nivå hade varit att föredra, men den är mycket fragmentarisk.

Luckorna i statistiken för olika år respektive olika regioner är relativt sett få. Statistiken för Norge, Liechtenstein och Schweiz är dock genomgående ofullständig, och som en konsekvens härav har dessa länder inte analyserats. Vidare är statistiken för Storbritannien och Grekland inte komplett. För flera av indikatorerna finns endast data för NUTSOnivån. Storbritannien har även gjort förändringar i sin NUTS-indelning för NUTS2-regionerna Inner London och Greater London, där fyra regioner blev fem. I Greklands fall finns ingen regional statistik för motorvägar och två regioner saknar genomgående regional statistik för flera av de valda indikatorerna. I Tyskland finns indikatorn för andelen av befolkningen som riskerar fattigdom och social exklusion (PEX) endast på nationell nivå (NUTS0), varför denna statistik används för alla regioner. I Österrikes fall finns denna indikator endast för 2011 och 2012. Statistiken för 2011 har använts för 2006 och statistiken för 2012 har använts för 2016. Troligen leder detta till att PEX överskattas för 2006 och underskattas för 2016. Andelen förnyelsebar energi (ENE) finns genomgående endast på nationell nivå, NUTSO. I tabellerna 2 och 3 listas tillgängligheten på statistik.

Tabell 2. Statistik, tillgänglighet per indikator och land 2006.

Table 2. Data availability per indicator and country 2006.

\begin{tabular}{|c|c|c|}
\hline & \begin{tabular}{|l|} 
Statistik \\
Data
\end{tabular} & $\begin{array}{l}\text { Källa } \\
\text { Source }\end{array}$ \\
\hline PPS & $\begin{array}{l}\text { NUTS 2, utom NUTS0 för IS, NO, CH: ingen statistik } \\
\text { NUTS 2, except NUTSO for IS, NO, CH: no data }\end{array}$ & Eurostat \\
\hline EMP & $\begin{array}{l}\text { Alla länder NUTS 2, utom DK och HR: statistik för 2007; SI, UK: statistik } 2010 \\
\text { All countries NUTS 2, except DK and HR: data for 2007; SI: data for 2010; UK data for } 2010 .\end{array}$ & Eurostat \\
\hline EDU & $\begin{array}{l}\text { NUTS 2, utom FR83 och FI20: extrapolade värden; PT20, PT30: NUTS } 0 \text { DK och HR: statistik 2007; } \\
\text { SI och UK: statistik } 2010 \\
\text { NUTS 2, except FR83 and FI20: extrapolated; PT20, PT30: NUTS 0 DK and HR: data for 2007; } \\
\text { SI and UK: data for } 2010\end{array}$ & Eurostat \\
\hline YOU & $\begin{array}{l}\text { NUTS 2, utom DK, HR: statistik för 2007; SI och UK: statistik för 2010; ITC1, AT11, PT15, DEB2, FR63, } \\
\text { FR68: NUTS 1; PT20, PT30: 2010; AT32, AT34: 2005; NL34, FI20 extrapolarede värden. } \\
\text { NUTS 2, except DK, HR: data for 2007; SI and UK: data for 2010; ITC1, AT11, PT15, DEB2, FR63, FR68: } \\
\text { NUTS 1; PT20, PT30: 2010; AT32, AT34: 2005; NL34, FI20 extrapolated }\end{array}$ & Eurostat \\
\hline
\end{tabular}




\begin{tabular}{|c|c|c|}
\hline YOU & $\begin{array}{l}\text { NUTS 2, utom DK, HR: statistik för 2007; SI och UK: statistik för 2010; ITC1, AT11, PT15, DEB2, FR63, } \\
\text { FR68: NUTS 1; PT20, PT30: 2010; AT32, AT34: 2005; NL34, FI20 extrapolarede värden. } \\
\text { NUTS 2, except DK, HR: data for 2007; SI and UK: data for 2010; ITC1, AT11, PT15, DEB2, FR63, FR68: } \\
\text { NUTS 1; PT20, PT30: 2010; AT32, AT34: 2005; NL34, FI20 extrapolated }\end{array}$ & Eurostat \\
\hline AGE & $\begin{array}{l}\text { NUTS 2, utom DK: statistik för 2007; DED4, DED5: statistik för } 2011 \\
\text { NUTS 2, except DK: data for 2007; DED4, DED5: data for } 2011\end{array}$ & Eurostat \\
\hline PEX & $\begin{array}{l}\text { NUTS 2, utom DE, FR, HR, PT, UK: statistik för NUTS 0; BE, EL, HU: statistik för NUTS 1; DK, RO: } \\
\text { NUTS 1; DK, RO: statistik för 2007; SI, FI1B, FI1C, FI1D, SE, CH: statistik för 2008; NL: } \\
\text { statistik för } 2011 \text { för NUTS 1; PL: statistik för 2012 för NUTS 1; AT: statistik för 2014 } \\
\text { NUTS 2, except DE, FR, HR, PT, UK: data for NUTS 0; BE, EL, HU: data for NUTS 1; DK, RO: } \\
\text { for NUTS 1; DK, RO: data for 2007; SI, FI1B, FI1C, FID, SE, CH: data for 2008; NL: } \\
\text { data for 2011 at NUTS 1; PL: data for } 2012 \text { at NUTS 1; AT: data for } 2014\end{array}$ & Eurostat \\
\hline ROA & $\begin{array}{l}\text { NUTS 2, utom DK: statistik för; DE11-14, DE21-27, DE71-73, DE91-94, DEB1-DEB3: statistik för 2005; } \\
\text { DEA1-DEA5: statistik för 2007; EL: NUTS0; UK: NUTS 1; ITG1, MT00: extrapolerad; SI: } \\
\text { statistik för NUTS 0; PT11, PT15, PT16, PT17, PT18, ITH2: statistik för NUTS 1 } \\
\text { NUTS 2, except DK: data for 2007; DE11-14, DE21-27, DE71-73, DE91-94, DEB1-DEB3: data for 2005; } \\
\text { DEA1-DEA5: data for 2007; EL: no data; UK: NUTS 1; ITG1, MT00: extrapolated; SI: } \\
\text { data for NUTS 0; PT11, PT15, PT16, PT17, PT18, ITH2: data at NUTS 1 }\end{array}$ & Eurostat \\
\hline INT & $\begin{array}{l}\text { NUTS 2, utom CZ, ES, PL, PT, FI20: statistik för 2007; BE, BG, DK, DE40, DE80, DED2, DEE0, DEG0, EE00, } \\
\text { IE01, IE02, HR03, HR04, CY, LU, HU, RO: statistik för 2008; LT, MT, SE: statistik för 2009; LV: statistik för } \\
\text { 2010; DE50: statistik för 2011; FR (utom FR19), FI1B, FI1C: extrapolerade värden; EL3, EL3, EL4: statistik för } \\
\text { NUTS } 1 \text { för 2006; EL5, EL6: extrapolerade värden; DE, PL, UK: statistik för NUTS 1; ITH5, ITI3: statistik för } \\
\text { NUTS } 1 \text { SI: statistik för NUTS 0; CH: ingen statistik } \\
\text { NUTS 2, except CZ, ES, PL, PT, FI20: data for 2007; BE, BG, DK, DE40, DE80, DED2, DEE0, DEG0, EE00, } \\
\text { IE01, IE02, HR03, HR04, CY, LU, HU, RO: data for 2008; LT, MT, SE: data for 2009; LV: data for 2010; DE50: } \\
\text { data for 2011; FR (except FR19), FI1B, FI1C: extrapolated; EL3, EL3, EL4: data at NUTS 1 for 2006; EL5, } \\
\text { EL6: no data; DE, PL, UK: data at NUTS 1; ITH5, ITI3: data at NUTS } 1 \text { SI: data at NUTS 0; CH: no data }\end{array}$ & Eurostat \\
\hline MED & $\begin{array}{l}\text { NUTS 2, utom IE, UK, SI: statistik för NUTS 0; DK: statistik för 2007; EL5, EL6: ingen statistik; MT: statistik } \\
\text { 2009; HR: statistik för 2010; BE: statistik för 2011 DE: statistik för NUTS 1; ES64, NL42, FI1B, FI1C, FI1D: } \\
\text { Extrapolerade värden; ITH5, ITI3: statistik för NUTS 0 } \\
\text { NUTS 2, except IE, UK, SI: data at NUTS 0; DK: data for 2007; EL5, EL6: no data; MT: data for 2009; HR: } \\
\text { data for 2010; BE: data for } 2011 \text { DE: data at NUTS 1; ES64, NL42, FI1B, FIIC, FI1D: } \\
\text { extrapolated; ITH5, ITI3: data at NUTS 0 }\end{array}$ & Eurostat \\
\hline ENE & $\begin{array}{l}\text { NUTS 0; NO, CH: ingen statistik } \\
\text { NUTS 0; NO, CH: no data }\end{array}$ & Eurostat \\
\hline
\end{tabular}

Tabell 3. Statistik, tillgänglighet per indikator och land 2016.

Table 3. Data availability per indicator and country 2006.

\begin{tabular}{|l|l|l|}
\hline & $\begin{array}{l}\text { Statistik } \\
\text { Data }\end{array}$ & $\begin{array}{l}\text { Källa } \\
\text { Source }\end{array}$ \\
\hline PPS & $\begin{array}{l}\text { NUTS 2 för 2015, utom IE och NO: statistik för 2014; IS: NUTS0, CH: ingen statistik } \\
\text { NUTS 2 for 2015, except IE and NO: data for 2014; IS, CH: no data }\end{array}$ & Eurostat \\
\hline EMP & $\begin{array}{l}\text { NUTS 2 } \\
\text { NUTS 2 }\end{array}$ & Eurostat \\
\hline EDU & $\begin{array}{l}\text { NUTS 2, utom PT20: extrapolerade värden } \\
\text { NUTS 2, except PT20: extrapolated }\end{array}$ & Eurostat \\
\hline
\end{tabular}




\begin{tabular}{|c|c|c|}
\hline YOU & $\begin{array}{l}\text { NUTS 2, utom DE22-DE24, DE26, DEB2, DED2, DED4, DED5, FR83, ITC2, AT11, AT34, PL43: } \\
\text { statistik för NUTS 1; DE73, PT20, PT30, UKD1, UKM6: 2014; DE50: 2011; BG31, DE25, DE80, DE93, FR63, } \\
\text { PL52, PT15, UKE2, UKK3: 2015 } \\
\text { NUTS 2, except DE22-DE24, DE26, DEB2, DED2, DED4, DED5, FR83, ITC2, AT11, AT34, PL43: } \\
\text { data at NUTS 1; DE73, PT20, PT30, UKD1, UKM6: 2014; DE50: 2011; BG31, DE25, DE80, DE93, FR63, } \\
\text { PL52, PT15, UKE2, UKK3: } 2015\end{array}$ & Eurostat \\
\hline AGE & $\begin{array}{l}\text { NUTS } 2 \\
\text { NUTS } 2\end{array}$ & Eurostat \\
\hline PEX & $\begin{array}{l}\text { NUTS 2, utom DE, FR, HR, PT, UK: NUTS 0; BE, EL, HU, NL, PL: NUTS 1; AT, IS: statistik för } 2015 \\
\text { NUTS 2, except DE, FR, HR, PT, UK: NUTS 0; BE, EL, HU, NL, PL: NUTS 1; AT, IS: data for } 2015\end{array}$ & Eurostat \\
\hline ROA & $\begin{array}{l}\text { NUTS } 2 \text { för 2015, utom ITH2, UK: NUTS 1; PT, EL: NUTS 0; DE11-14, DE91-94: } \\
\text { statistik för 2009; DE21-27, DE71-73, DEA1-A5, DEB1-B3, DED2, DED4, DED5: } \\
\text { statistik för 2010; SI, NO: statistik för 2012; IT, FI, CH: statistik för 2014; MT: extrapolerade värden } \\
\text { NUTS } 2 \text { for 2015, except ITH2, UK: NUTS 1; PT: NUTS 0; EL: no data; DE11-14, DE91-94: } \\
\text { data for 2009; DE21-27, DE71-73, DEA1-A5, DEB1-B3, DED2, DED4, DED5: } \\
\text { data for 2010; SI, NO: data for 2012; IT, FI, CH: data for 2014; MT: extrapolated }\end{array}$ & Eurostat \\
\hline INT & $\begin{array}{l}\text { NUTS2 för 2017, utom PT: 2016; DE, EL, PL, UK: statistik för NUTS 1; FI20: extrapolerade värden } \\
\text { NUTS } 2 \text { for 2017, except PT: data for 2016; DE, EL, PL, UK: data at NUTS 1; FI20: extrapolated }\end{array}$ & Eurostat \\
\hline MED & $\begin{array}{l}\text { NUTS 2, utom CZ: 2013; DK, FR, SK, FI, SE: 2014; IE, SI, UK: NUTS 0; DE: NUTS 1; } \\
\text { ES64, EL51-54, EL61-64: extrapolerade värden } \\
\text { NUTS 2, except CZ: 2013; DK, FR, SK, FI, SE: 2014; IE, SI, UK: NUTS 0; DE: NUTS 1; } \\
\text { ES64: extrapolated; EL51-54, EL61-64: no data. }\end{array}$ & Eurostat \\
\hline ENE & $\begin{array}{l}\text { NUTS 0; NO, CH: ingen statistik } \\
\text { NUTS 0; NO, CH: no data }\end{array}$ & Eurostat \\
\hline
\end{tabular}

Reliabilitet betraktas som mätningens konsistens och replikerbarhet. För att öka konsistensen på vad som mäts i detta index har harmoniserad statistik från Eurostat använts, och de approximationer som gjorts för t.ex. andelen av befolkningen i risk för fattigdom och social exklusion i Tyskland och Österrike kommer inte att skapa systemfel i uppskattningarna. Tabellerna 2 och 3 innehåller detaljerad information över den använda statistiken och hur inkomplett statistik hanterats. Liechtenstein, Norge och Schweiz har uteslutits på grund av att statistiken för dessa länder uppvisar för många brister.

\section{Att konstruera ett index}

Det index som konstrueras här, Territorial Cohesion Development Index (TCDI), bygger på samma metodologi som används för FN:s Human Development Index (HDI). Precis som i HDI, kommer de mål som anges som policymålsättningar att användas som riktvärden. Minimi- och maximivärden beräknas för att transformera olika enheter till ett index på en skala från 0 till 1 (UNDP 2016b).
Komponentindikatorerna standardiseras sedan med utgångspunkt från de mål som formuleras i policydokumenten (Ekvation 1a, 1b). I de fall målen i policydokumenten uppnås (se Tabell 1) kommer indikatorn anta värdet 1 .

Det är värt att notera att indikatorn för PEX har ett maxvärde som är mindre än 100 . Inte i något land i världen lever $100 \%$ av befolkningen i fattigdom - inte ens i länder som exempelvis Afghanistan, Irak eller Somalia (World Bank 2018). Därför har maxvärdet för PEX satts till lägre än 100 procent.

När minimi- och maximivärdena för en indikator beräknats kan ett delindex Ix beräknas på samma sätt som i HDI (UNDP 2016b) - se ekvation 1a. För indikatorer som uppnått policymålsättningen $\mathrm{x}_{\max }$, ersätts nämnaren med det definierade målvärdet.

$$
I_{x}=\frac{x_{\text {actual }}-x_{\min }}{x_{\max }-x_{\min }}
$$

För indikatorer som andelen av befolkningen i åldern 65+ år, andelen av befolkningen i risk för 
fattigdom och social exklusion samt ungdomsarbetslösheten är ett högt värde inte positivt. Detta kontrolleras för genom att delindexet inverteras för dessa indikatorer på det sätt som visas i ekvation $1 b$.

$$
I_{x}=1-\frac{x_{a c t u a l}-x_{\min }}{x_{\max }-x_{\min }}
$$

TCDI beräknas sedan som det aritmetiska medelvärdet av de olika delindexen ${ }^{1}$ :

$$
T C D I=\frac{\left(I_{x}+I_{y}+\cdots+I_{n}\right)}{n}
$$

Förändringar över tid i TCDI beräknas enligt följande:

$$
\Delta T C D I=T C D I_{(t+1)}-T C D I_{t}
$$

HDI är känt för att ge robusta resultat. Givet att de använda indikatorerna mäter det de förmodas mäta (Marques da Costa et al. 2013), kan man anta att TDCI även producerar robusta resultat på det sätt HDI gör.

\section{Resultat}

Länder såsom Lettland, Polen, Tjeckien, Slovakien, Ungern, Rumänien, Bulgarien och Grekland samt södra Italien får relativt sett låga värden i TCDI. Perifera länder såsom Irland, Island, Sverige, Finland, Estland, Litauen och Cypern får däremot relativt sett höga värden i TCDI. Det gör även regioner i Tyskland, Frankrike, nordöstra Spanien, Nederländerna, Luxembourg och London. På det hela taget bekräftar TDCI den bild de flesta har av sammahållningspolitiken i EU 2006 (Figur 1).

Över lag visar värdena för 2016 att länder såsom Lettland, Polen, Tjeckien, Slovakien och Ungern gynnats av ECP. Detta är ett förväntat utfall. Att de

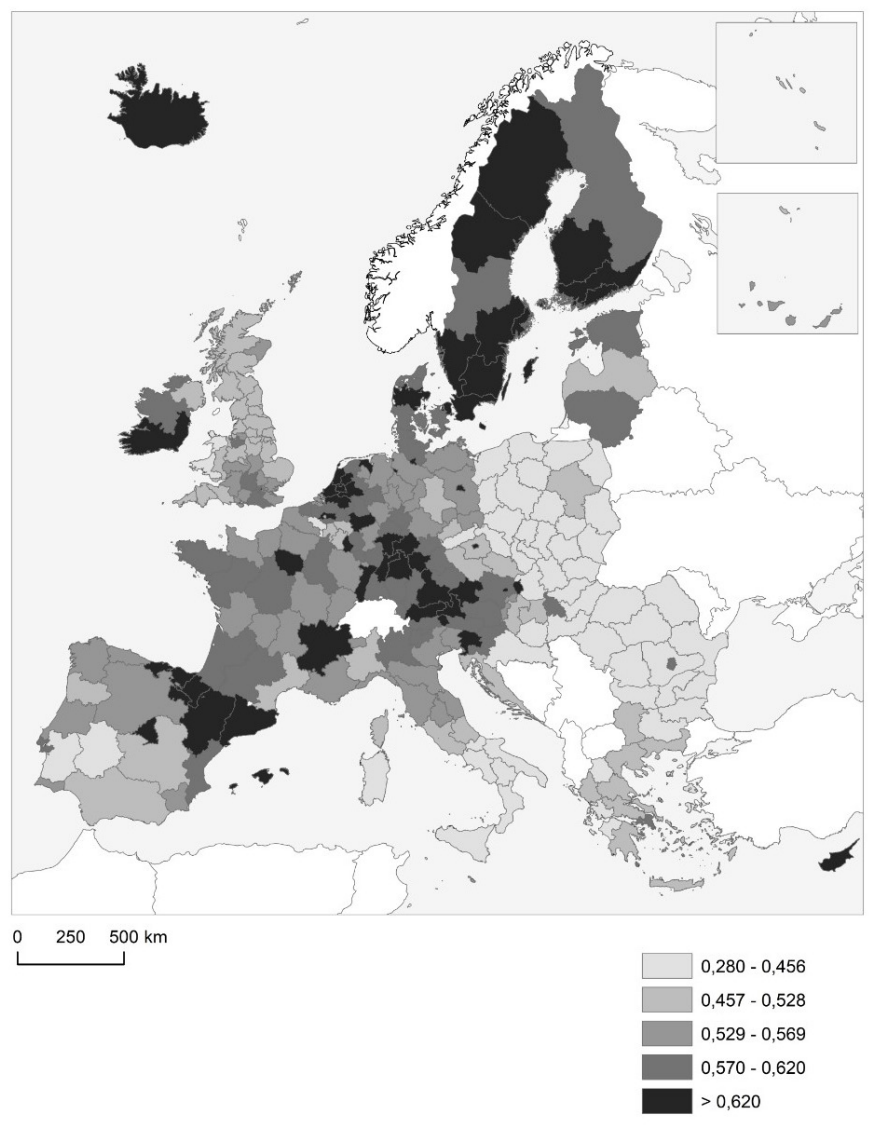

Figur 1. Territorial Cohesion Development Index (TCDI) resultat 2006 (kvintiler).

\section{Figure 1. Territorial Cohesion Development Index (TCDI) result 2006 (quintiles).}

1 Eftersom visa av indikatorerna kan anta värdet noll kan inte det geometriska medelvärdet användas, vilket gör att det aritmetiska medelvärdet måste användas i stället. 


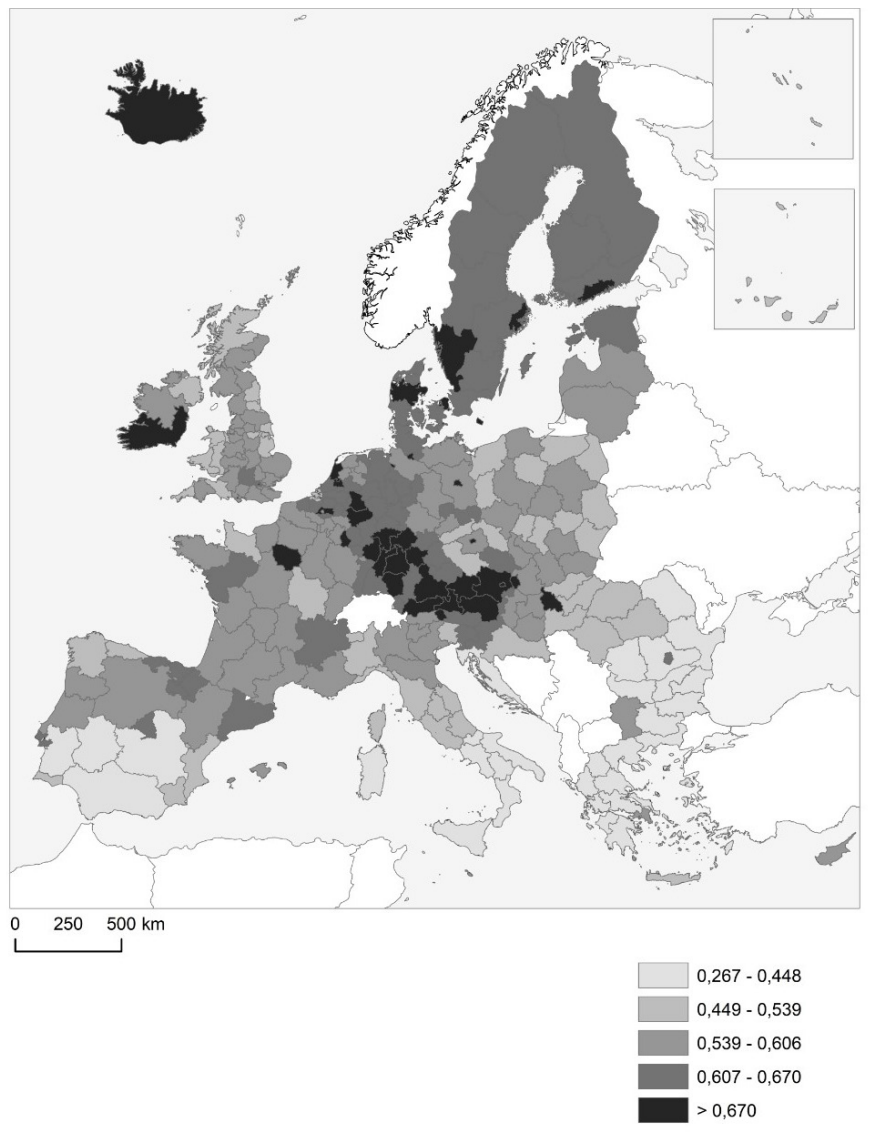

Figur 2. Territorial Cohesion Development Index (TCDI) resultat 2016 (kvintiler).

Figure 2. Territorial
Cohesion Development
Index (TCDI) result
2016 (quintiles).

Figure 2. Territorial Index (TCDI) result 2016 (quintiles). nordiska länderna, Estland och Cypern också har ett högt TDCI-resultat är inte heller förvånande. Mot bakgrund av den finansiella krisen som särskilt kom att drabba länder som Grekland, Spanien, Portugal, Italien och Irland är det inte förvånande att värdena i TCDI minskat. Snarare är de relativt höga värdena för de irländska regionerna 2016 förvånande (Figur 2).

När värdena för TCDI jämförs mellan 2006 och 2016 uppträder intressanta resultat. Vid första anblicken är resultaten de förväntade: länder såsom Lettland, Polen, Tjeckien, Slovakien, Ungern, Rumänien och Bulgarien har alla ökat sina värden i TCDI markant. Ett uttalat mål med ECP är att minska klyftorna mellan regioner i de nya och gamla medlemsländerna, och att denna process är pågående framkommer av resultaten i TCDI. De kraftiga förbättringarna i TCDI för Danmark, Österrike, delar av Tyskland och Belgien är däremot förvånande (Figur 3). Att den hårdföra krispolitik som antogs i Grekland, Spanien, Italien och Island kunde leda till större klyftor sett ur ett sammanhållningsperspektiv är inte förvånande, och detta är något som framkommer av resultaten i Figur 3. Däremot är den negativa utveckling som framkommer i större delen av Finland och Sverige samt delar av Nederländerna och Frankrike förvånande. Även om Sverige och Finland har höga värden i TCDI både för 2006 och 2016, har de förlorat relativt sett 2016. Tyska storstäder (Hamburg, Bremen och Berlin) framstår som klara vinnare.

Medan fem regioner i Europas periferi (Stockholm, Madrid, Island, Helsingfors samt södra och östra Irland) var bland de 10 regioner som hade de högsta resultaten 2006 fanns det bara två kvar på top-10-listan 2016. Åtta av de 10 regioner som hade högst värde 2016 är centraleuropeiska storstadsregioner (Tabell 4). Detta indikerar inte bara att ECP gynnat storstadsområden efter krisen 2009, utan även att det skett en förskjutning från länder i EU:s utkanter till förmån för Centraleuropa.

När de 10 regioner som presterade sämst i TCDI 2006 och 2016 analyseras framkommer dramatiska förändringar mellan de båda åren. Medan åtta av de 10 regioner som presterade sämst 2006 var be- 


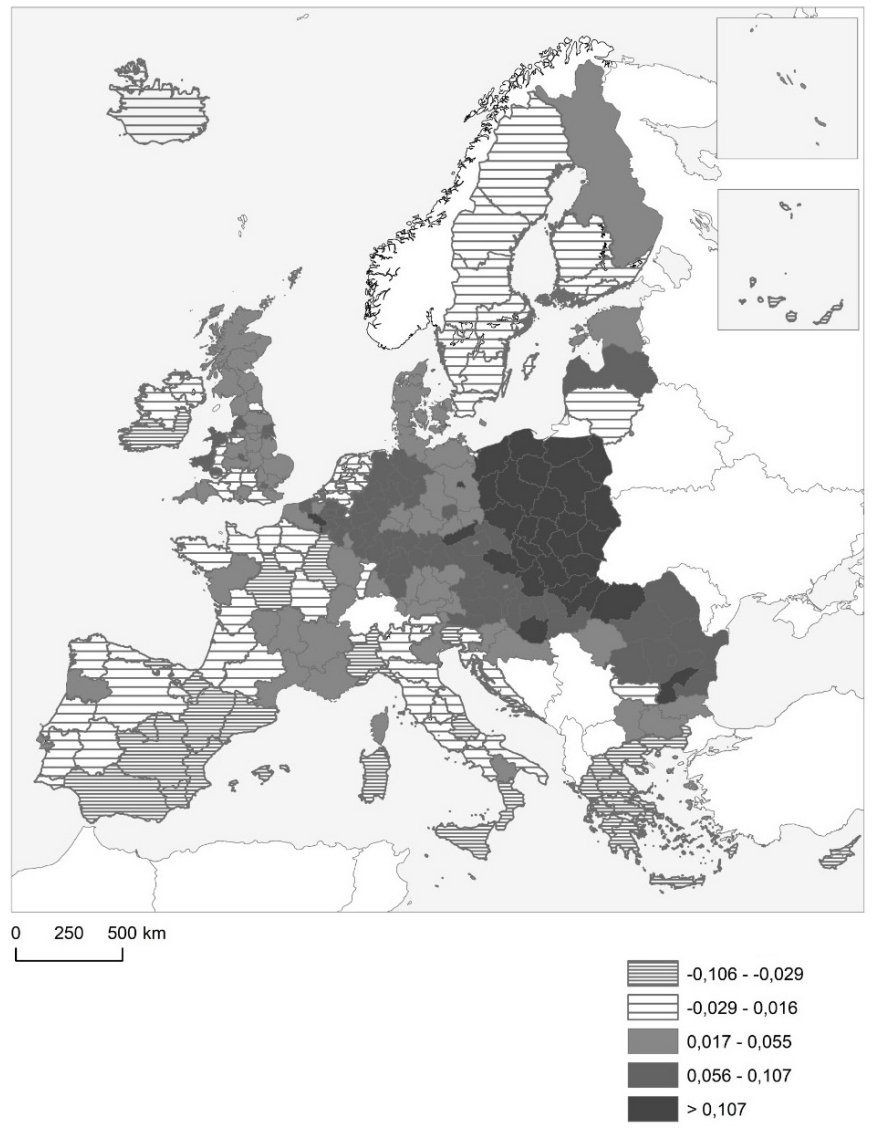

Figur 3. Skillnader i Territorial Cohesion Development Index (TCDI) mellan 2006 och 2016 (kvintiler).

Figure 3. Changes in the Territorial Cohesion Development Index (TCDI) between 2006 and 2016 (quintiles).

Tabell 4. De 10 NUTS 2 regioner med högst TCDI-värden 2006 och 2016.

Table 4. The 10 NUTS2 regions with highest TCDI scores in 2006 and 2016.

\begin{tabular}{|l|l|l|c|}
\hline \multicolumn{2}{|c|}{ 2006 } & \multicolumn{2}{c|}{ 2016 } \\
\hline RCgion & 0,75746 & DE60 - Hamburg & $\begin{array}{l}\text { TCDI-värde / } \\
\text { TCDI score }\end{array}$ \\
\hline SE11 - Stockholm & 0,74111 & DE50 - Bremen & 0,79031286 \\
\hline ES30 - Comunidad de Madrid & 0,73626 & SK01 - Bratislavský kraj & 0,749437728 \\
\hline NL31 - Utrecht & 0,73498 & DK01 - Hovedstaden & 0,74881797 \\
\hline IS00 - Ísland & 0,72953 & DE30 - Berlin & 0,742687223 \\
\hline FI1B - Helsinki-Uusimaa & 0,71373 & SE11 - Stockholm & 0,739828922 \\
\hline DK01 - Hovedstaden & 0,71125 & LU00 - Luxembourg & 0,729426031 \\
\hline LU00 - Luxembourg & 0,70916 & AT13 - Wien & 0,72482085 \\
\hline IE02 - Southern and Eastern & 0,70222 & FI1B - Helsinki-Uusimaa & 0,722394274 \\
\hline Ireland & 0,69930 & DE71 - Darmstadt & 0,721028542 \\
\hline DE60 - Hamburg & & & \\
\hline NL32 - Noord-Holland & & & Region \\
\hline
\end{tabular}


184 Rauhut \& Costa EU:s sammanhållningspolitik och regionala...

Tabell 5. De 10 NUTS 2 regioner med lägst TCDI-värde 2006 och 2016.

Table 5. The 10 NUTS2 regions with lowest TCDI scores in 2006 and 2016.

\begin{tabular}{|l|l|l|l|}
\hline \multicolumn{2}{|c|}{ 2006 } & \multicolumn{1}{c|}{ TC16 } \\
\hline Region & 0,33564 & BG40̈2 - Yuzhen tsentralen & $\begin{array}{l}\text { TCDI-värde } \\
\text { TCDI score }\end{array}$ \\
\hline PL32 - Podkarpackie & 0,33053 & EL64 - Sterea Ellada & 0,388054887 \\
\hline ITG1 - Sicilia & 0,32929 & RO22 - Sud-Est & 0,387497036 \\
\hline ITF4 - Puglia & 0,32713 & RO31 - Sud - Muntenia & 0,370837283 \\
\hline RO21 - Nord-Est & 0,32054 & ITG2 - Sardegna & 0,356947684 \\
\hline PL33 - Swietokrzyskie & 0,31962 & ITF4 - Puglia & 0,327151881 \\
\hline RO41 - Sud-Vest Oltenia & 0,31300 & ITF3 - Campania & 0,323387978 \\
\hline RO22 - Sud-Est & 0,29795 & ITF6 - Calabria & 0,291951828 \\
\hline RO31 - Sud - Muntenia & 0,28566 & BG31 - Severozapaden & 0,278167154 \\
\hline BG32 - Severen tsentralen & 0,28006 & ITG1 - Sicilia & 0,267486101 \\
\hline BG31 - Severozapaden & & &
\end{tabular}

lägna i de nya medlemsländerna Polen, Rumänien och Bulgarien, låg endast fyra av de 10 regionerna som hade sämst TCDI-värde 2016 i de under 2000-talet tillkomna medlemsländerna. Fem av de lågpresterande regionerna var italienska och en var grekisk (Tabell 5). Detta indikerar en förskjutning av problem med sammanhållningen från att ha varit ett problem som främst hörde samman med de nya medlemsländerna till att bli ett problem i de gamla medlemsländerna.

Analyseras förändringen mellan 2006 och 2016 framkommer denna förskjutning av sammanhållningsproblem - från nya medlemsländer till de gamla - än tydligare. De 10 regioner som förbättrade sina TCDI-värden mest mellan de båda åren ligger alla i de nya medlemsländerna och de är även belägna i Centraleuropa. Samtliga regioner som uppvisar de kraftigaste försämringarna i TCDI-resultat mellan de båda analyserade åren ligger i de gamla medlemsländerna Grekland, Italien och Spanien. Det är även värt att notera att samtliga de stora "förlorar"-regionerna är belägna i EU:s periferi (Tabell 6).

\section{Diskussion}

Om man analyserar de regioner som tjänat mest på ECP 2006-2016 med hjälp av det index som konstruerats i denna studie, TCDI, så framkommer det att dessa regioner har en del gemensamt. Enligt den regionala klassificering som ESPON (2011) gör framkommer det att 10 av de 20 regionerna med de högsta TCDI-värdena 2006 är belägna i EU:s periferi (fyra svenska regioner, tre spanska, en finländsk, en irländsk och Island), 12 är stadsregioner (Helsinki-Uusimaa; Stockholm och Västsverige; Île de France; Wien; Luxembourg; Hovedstaden; Southern and Eastern Ireland; Comunidad de Madrid och Cataluña; Utrecht, Noord-Holland och ZuidHolland; Oberbayern, Bremen och Hamburg), och tre är rurala regioner (Island, Östra Mellansverige och Comunidad Foral de Navarra). År 2016 ligger endast tre av de 20 regionerna med högst TCDIvärde i EU:s periferi (Stockholm, Island och Helsinki-Uusimaa), två är rurala (Salzburg och Island), medan övriga är städer eller storstadsregioner belägna i Centraleuropa (Bremen, Hamburg, Berlin, Darmstadt, Düsseldorf, Oberbayern, Köln, Stuttgart, Karlsruhe, Bratislavský kraj, Hovedstaden, Luxembourg, Wien, Inner London West, VlaamsBrabant och Utrecht). Det är även värt att notera att nio av de 20 regionerna med högst TCDI-värden 2016 är tyska, medan endast två var det 2006.

De 20 regioner som uppvisar den kraftigaste förbättringen av sina TCDI-värden är alla belägna $\mathrm{i}$ Centraleuropa. Dessa regioner klassas som städer, storstadsregioner eller regioner i närheten av storstäder och som genomgår en strukturell omvandling (av vilka 12 är polska, två slovakiska, två tjeckiska och en är rumänsk). Endast tre (polska) regioner 
Tabell 6. De 10 NUTS 2 regioner med högst positiva respektive negativa förändring av TCDI-värdet mellan 2006 och 2016.

Table 6. Highest positive and negative changes in TCDI scores for NUTS 2 regions between 2006 and 2016.

\begin{tabular}{|c|c|c|c|}
\hline \multicolumn{2}{|c|}{$\begin{array}{l}\text { Störst positiv förändring } \\
\text { Highest positive change }\end{array}$} & \multicolumn{2}{|c|}{$\begin{array}{l}\text { Störst negativ förändring } \\
\text { Highest negative change }\end{array}$} \\
\hline Region & $\begin{array}{l}\text { TCDI-värde } \\
\text { TCDI score }\end{array}$ & Region & $\begin{array}{l}\text { TCDI-värde } \\
\text { TCDI score }\end{array}$ \\
\hline PL51 - Dolnoslaskie & 0,18126 & ITG1 - Sicilia & $-0,06304$ \\
\hline $\begin{array}{l}\text { SK04 - Východné } \\
\text { Slovensko }\end{array}$ & 0,17283 & ES53 - Illes Balears & $-0,06967$ \\
\hline $\begin{array}{l}\text { PL61 - Kujawsko- } \\
\text { Pomorskie }\end{array}$ & 0,17025 & ES30 - Comunidad de Madrid & $-0,07052$ \\
\hline PL43 - Lubuskie & 0,16952 & EL64 - Sterea Ellada & $-0,07676$ \\
\hline PL33 - Swietokrzyskie & 0,16522 & ES42 - Castilla-la Mancha & $-0,07724$ \\
\hline PL41 - Wielkopolskie & 0,16431 & EL61 - Thessalia & $-0,08286$ \\
\hline PL11 - Lódzkie & 0,16239 & $\begin{array}{l}\text { ES52 - Comunidad } \\
\text { Valenciana }\end{array}$ & $-0,08785$ \\
\hline CZ08 - Moravskoslezsko & 0,16001 & ES61 - Andalucía & $-0,09715$ \\
\hline PL63 - Pomorskie & 0,15424 & ES70 - Canarias (ES) & $-0,09778$ \\
\hline CZ06 - Jihovýchod & 0,15136 & $\begin{array}{l}\text { ES64 - Ciudad Autónoma de } \\
\text { Melilla (ES) }\end{array}$ & $-0,10647$ \\
\hline
\end{tabular}

klassificeras som mellanliggande i förhållande till större städer eller rurala. Hela 15 av de 20 regioner som ökat sina TCDI-värden mest är belägna i Polen.

Även de regioner som uppvisar de största negativa förändringarna i TCDI-resultat mellan de analyserade åren uppvisar stora likheter. Bland regionerna som uppvisade lägst TCDI-värde 2006 var en spansk, en ungersk, sju polska, tre bulgariska, fyra rumänska och fyra italienska. En av dessa regioner är geografiskt belägen i Afrika (Ciudad Autónoma de Ceuta), tre är urbana (Severozapaden, Severen tsentralen och Ciudad Autónoma de Ceuta), och 11 är belägna i utkanten av EU (fyra i Rumänien, en i Ungern, fyra i Italien och två i Bulgarien). Vidare klassificeras 10 regioner som mellanliggande i förhållande till större städer och avindustrialiserade (varav fem ligger i Polen, tre i Bulgarien, en i Ungern och en i Rumänien), och sju regioner klassificeras som rurala och mellanliggande i förhållande till stora städer (tre rumänska regioner, tre italienska och en polsk). En region är mellanliggande i förhållande till stora städer och urban (Campania).

Samtliga regioner med lägst TCDI-värde 2016 är belägna i EU:s utkanter. En är ren huvudstadsregion (Severozapaden i Bulgarien) och två är mellanliggande till stora städer och urbana (Andalucía och
Campania). Hela 12 av de 20 med lägst TCDI-värde klassificeras som mellanliggande till stora städer och rurala (fem ligger i Grekland, fem i Italien, och Rumänien), och fem är mellanliggande till stora städer och genomgår en avindustrialisering (tre bulgariska regioner, en grekisk och en rumänsk). Av de 20 regionerna med lägst TCDI-värden 2016 är 13 belägna i EU:s gamla medlemsländer.

Bland de regioner som uppvisar de största försämringarna i TCDI-värde mellan 2006 och 2016 är endast en belägen i Centraleuropa: den italienska regionen Ligurien, en region som klassificeras som bergsregion och urban. Övriga 19 regioner är belägna i EU:s utkanter. Två regioner är huvudstadsregioner (Comunidad de Madrid och Cypern), en är i huvudsak urban och genomgår en industriell omvandling (Cataluña), och en är mellanliggande till större städer och urban (Andalucía). 12 regioner är mellanliggande till större städer och rurala (varav fem ligger i Grekland, tre i Italien och fyra i Spanien), och tre (en i Grekland och två i Spanien) är mellanliggande till större städer och genomgår en industriell omvandling. Alla utom Cypern är belägna i de gamla EU15-länderna.

Varför vissa regioner vann på ECP under den analyserade perioden medan andra förlorade hör sam- 
man med bland annat ekonomisk struktur och diversifiering, hållbarhet vid kriser och åtstramningspolitik. Den globala finanskrisen 2008-2009 drabbade olika regioner på olika sätt och olika mycket (Hadjimichalis 2011). Den förändring av EU:s sammanhållningspolitik som ägde rum efter krisen 2008-2009 - från sammanhållning till konkurrens och från svaga regioner till storstäder - indikerar ett större fokus på städer och storstadsregioner (Faludi m.fl. 2015). Vidare innebär den platsbaserade strategin som följde på rapporten av Barca (2009), att lokala aktörer får ett större ansvar för en territorialisering av de ekonomiska och sociala agendorna, vilket innebär att de utmaningar perifera regioner brottas med lämnas åt dem att själv lösa. Tyvärr har dessa aktörer sällan de instrument och resurser som krävs för att hantera dessa utmaningar (Solly 2016; Gruber m.fl. 2019; Isola m.fl. 2017; Clifton m.fl. 2015). Till detta kan man lägga empiriska studier som visar på att förutsättningarna för en framgångsrik platsbaserad politik sällan uppfylls (Partridge m.fl. 2015). Fortfarande bör politiken anpassas efter varje lands specifika egenskaper och vara lokalt baserade (Camagni \& Capello 2015a), och detta återspeglar frågan om i vilken utsträckning policyn Community Led Local Development faktiskt kan stimulera utvecklingen i en önskad riktning (Servillo 2019). För att uppnå en mer balanserad utveckling krävs att städer utanför tillväxtregionerna stimuleras (Medeiros \& Rauhut 2020).

\section{Sammanfattning och slutsatser}

Syftet med denna studie är att analysera den europeiska sammanhållningspolitiken och dess implikationer på NUTS2-nivå för perioden 2006-2016, det vill säga före och efter den finansiella krisen 2009. Tre frågor ställdes.

(1) Angående vilken typ av regioner som gynnats av ECP under den analyserade perioden, har främst stora städer och storstadsregioner kommit att gynnas. Regioner i Tyskland och Polen framstår som de stora vinnarna. Från att ha presterat väl i TCDI 2006 kom de tyska regionerna att prestera mycket väl i TCDI 2016. De polska regionerna uppvisar störst ökning av sina TCDI-värden mellan de analyserade åren - från att ha haft låga TCDI-värden 2006 ökade de till medelmåttiga 2016.

(2) Även de regioner som har förlorat på ECP under den analyserade perioden uppvisar likheter. En sådan likhet är att de är belägna i utkanten av EU, långt från det polycentriska och tätbefolkade Centraleuropa. Generellt sett talar vi om regioner som är mellanliggande i förhållande till städer och som antingen är rurala eller brottas med en negativ industriell omvandling. Dessa regioner är främst belägna i Italien, Spanien och Grekland. Det är dock inte bara grekiska, spanska och italienska regioner som brottas med en negativ utveckling sett ur ett sammanhållningsperspektiv. Fyra av fem finska regioner, sju av åtta svenska regioner, båda de irländska regionerna, och flera franska och nederländska regioner uppvisar en negativ TCDIutveckling. Dessa länder hade höga TCDI-värden 2006, men 2016 hade de sjunkit.

(3) Förändringen av EU:s sammanhållningspolitik efter krisen 2008-2009 - från sammanhållning till konkurrens och från svaga regioner till storstäder - har resulterat i en annan, mindre diskuterad konsekvens. Före förändringen av ECP åtnjöt länder och regioner i utkanten av EU stöd av olika slag; efter omläggningen av ECP har dessa länder och regioner förlorat sitt stöd till förmån för städer och storstadsregioner i Centraleuropa. Med andra ord, stödet har omallokerats från periferi till centrum.

Den ekonomiska teori som ECP efter 2009 bygger på, "growth pole theory", har sina rötter i de tankar den franske ekonomen Perroux formulerade 1949. Han ville försöka stimulera återuppbyggnaden av den franska tillverkningsindustrin och dess regionala försörjningskedjor efter andra världskriget. Teorin vidareutvecklades av Hirschman under 1950-talet när han försökte skapa strategier för att industrialisera jordbruksekonomierna i Sydostasien. De flesta av antagnadena i "growth pole theory" uppfylls inte i den kontext som ges av EU:s post-industriella serviceekonomi med globala försörjningskedjor (Rauhut \& Humer 2020). En ekonomisk teori, som formulerades för villkor fundamentalt annorlunda än den kontext de nu implementeras i, måste ifrågasättas. Resultaten från TCDI indikerar detta politikmisslyckande.

Några förslag på framtida forskning kan även ges. Studier där TCDI används för att analysera utvecklingen på NUTS3-nivå bör genomföras, antingen för de NUTS3-regioner inom EU där statistik finns tillgänglig eller för ett fåtal länder med god statistik. Eventuellt kan utvecklingen analyseras utifrån teori om spatial justice. Vidare är det möjligt att analysera regioner som klassificerats på samma sätt enligt den indelning ESPON (2011) gjort. Man kan även analysera utvecklingen utifrån ett centrum/periferi-perspektiv med hjälp av TCDI. Även longitudinella studier av TCDI behövs. I denna studie har endast två år analyserats, men det är fullt möjligt att beräkna TCDI på regional nivå för ett land sedan 1990-talets början. På så sätt kan man kontrollera för strukturkriser och konjunkturella svängningar som påverkar TCDI.

Slutsatsen av denna studie är att TCDI visar att centraleuropeiska regioner, huvudsakligen städer och storstadsregioner, vunnit på ECP 2006-2016, 
medan regioner i utkanten av EU, och då särskilt regioner som karaktäriseras av att vara mellanliggande i förhållande till städer och som antingen är rural eller brottas med en negativ industriell omvandling, har förlorat på ECP. Många av dessa regioner har erfarit högre arbetslöshet och försämrad välfärd (Essletzbichler m.fl. 2018; Fratesi \& RodríguezPose 2016; Lobao m.fl. 2018). Det är rationellt av väljarkåren att vända sig mot en sådan utveckling och de protester som förekommit har starka territoriella rötter (Rodríguez-Pose 2018), då den förda politiken inte längre kan skapa möjligheter för människor som bor på dessa platser (Essletzbichler m.fl. 2018; Dijkstra m.fl. 2020). När det upplevda sociala kontraktet mellan invånarna i förlorarregionerna och den centrala statsmakten i dessa länder börjar lösas upp, kan väljarkåren vända sig mot centralmakten och EU (Rauhut 2018). Om inte ECP åter börjar fokusera på sammanhållning riskerar EU att ifrågasättas av invånarna utanför det gynnade Centraleuropa. En sådan utveckling kan äventyra inte bara ECP, utan även EU i sig. Förhoppningsvis tar den nya sammahållningspolitiken hänsyn till detta. Om inte, så kommer vi att erfara fler länder som vill lämna EU.

\section{Tillkännagivande}

Denna studie har delvis finansierat genom Horizon 2020-projektet 'Resituating the Local in Cohesion and Territorial Development' (RELOCAL), Grant Agreement nr. 7270970.

\section{Litteratur}

Bachtler, J., Begg, I., Charles, D. \& Polverari, L. (2016) The long-term effectiveness of EU Cohesion Policy: assessing the achievements of the ERDF, 1989-2012. I: Bachtler J., Berkowitz P., Hardy S. \& Muravska T. (eds.) EU Cohesion Policy: Reassessing performance and direction. Routledge, London.

Bachtler, J., Polverari, L., Oraz, E. H., Clement, K. \& Tödtling-Schönhofer, H. (2009) Ex post evaluation of cohesion policy programmes 2000-2006 co-financed by the ERDF (objective 1 and 2): Management and implementation systems for cohesion policy. Report to the Commission of the European Communities, Brussels.

Bakucs, Z., Fertő, I., Varga, A. \& Benedek, Z. (2018) Impact of European Union development subsidies on Hungarian regions. European Planning Studies 28(6) 1121-1136. https://doi.org/10.1080/09654313.2018.1437394

Barca, F. (2009) An Agenda for a Reformed Cohesion Policy, A Place Based Approach to Meeting European Union Challenges and Expectations. Indepen- dent report prepared at the request of Danuta Hubner, Commissioner for Regional Policy. <https:// ec.europa.eu/regional policy/archive/policy/future/ pdf/report_barca_v0306.pdfs 26.8.2020.

Becker, S. O., Egger, P. H. \& von Ehrlich, M. (2012) Too much of a good thing? On the growth effects of the EU's regional policy. European Economic Review 56(4) 648-668. https://doi.org/10.1016/j.euroecorev.2012.03.001

Becker, S. O., Egger, P. H. \& von Ehrlich, M. (2018) Effects of EU Regional Policy: 1989-2013. Regional Science and Urban Economics 69 143-152. https://doi.org/10.1016/j.regsciurbeco.2017.12.001

Böhme, K., Douchet, P., Komornicki, T., Zaucha, J. \& Swiatek, D. (2011) How to strengthen the territorial dimension of 'Europe 2020' and the Cohesion Policy. $A$ report prepared at the request of the Polish EU Presidency of the Council of the European Union. Ministry of Regional Development, Warsaw.

Bradley, J. (2006) Evaluating the impact of European Union Cohesion policy in less-developed countries and regions. Regional Studies 40(2) 189-200. https://doi.org/10.1080/00343400600600512

Camagni, R. \& Capello, R. (2015a) Rationale and design of EU cohesion policies in a period of crisis. Regional Science Policy and Practice 7(1) 25-49. https://doi.org/10.1111/rsp3.12047

Camagni, R. \& Capello, R. (2015b) SecondRank City Dynamics: Theoretical Interpretations Behind Their Growth Potentials. European Planning Studies 23(6) 1041-1053. https://doi.org/10.1080/09654313.2014.904994

Capello, R., Caragliu, A. \& Fratesi, U. (2015) Spatial heterogeneity in the costs of the economic crisis in Europe: are cities sources of regional resilience? Journal of Economic Geography 15 (5) 951-972. https://doi.org/10.1093/jeg/lbu053

Cappelen, A., Castellacci, F., Fagerberg, J. \& Verspagen, B. (2003) The Impact of EU Regional Support on Growth and Convergence in the European Union. Journal of Common Market Studies 41(4) 621-644. https://doi.org/10.1111/1468-5965.00438

CEC (2001) Unity, Solidarity, Diversity for Europe, its People and its Territory, Second Report on Economic and Social Cohesion. European Commission, Brussels. CEC (2004) A New Partnership for Cohesion: Convergence, Competitiveness, Cooperation. Third Report on Economic and Social Cohesion. Office for Official Publications of the European Communities, Luxembourg.

CEC (2007) Growing Regions, Growing Europe. Fourth report on economic and social cohesion. Luxembourg: Office for Official Publications of the European Communities.

CEC (2010a) Europe 2020 - A strategy for smart, sustainable and inclusive growth. COM (2010) 2020 
final. <https://ec.europa.eu/eu2020/pdf/COMPLET\%20EN\%20BARROSO\%20\%20\%20007\%20 -\%20Europe\%202020\%20-\%20EN\%20version.pds 26.8.2020.

CEC (2010b) Investing in Europe's Future. Fifth Report in Economic, Social and Territorial Cohesion. Publications Office of the European Union, Luxembourg. <https://ec.europa.eu/regional policy/ sources/docofficlofficial/reports/cohesion $5 / p d f / 5 \mathrm{cr}$ en.pdf> 26.8.2020.

CEC (2014) Investment for jobs and growth. Sixth report on economic, social and territorial cohesion. Luxembourg: Publications Office of the European Union. <https://ec.europa.eu/regional policy/sources/docoffic/official/reports/cohesion6/6cr en.pdfs 26.8.2020.

CEC (2016) Investing in Europe's Youth. COM(2016) 940 final. <https://ec.europa.eu/transparency/regdoc/ rep/1/2016/EN/COM-2016-940-F1-EN-MAIN.PDF> 26.8.2020.

CEC (2017) My Region, My Europe, Our Future. Seventh report on economic, social and territorial cohesion. Publications Office of the European Union, Luxembourg. <https://ec.europa.eu/regional policy/ sources/docoffic/official/reports/cohesion $7 / 7 \mathrm{cr}$.pdfs 26.8.2020.

Clifton, J., Diaz-Fuentes, D. \& Fernandez-Gutíerrez, M. (2015) Public Infrastructure Services in the European Union: Challenges for Territorial Cohesion. Regional Studies 50(2) 358-373. https://doi.org/10.1080/00343404.2015.1044958

Dijkstra, L. \& Athanasoglou, S. (2015) The Europe 2020 index: The progress of EU countries, regions and cities. Regional Focus Working Paper 01/2015. $<$ https://ec.europa.eu/regional policy/sources/docgener/focus $/ 201501$ europe 2020 index.pdf> 26.8.2020.

Dijkstra, L., Garcilazo, E. \& McCann, P. (2013) The economic performance of European cities and city regions: Myths and realities. European Planning Studies 21(3) 334-354. https://doi.org/10.1080/09654313.2012.716245

Dijkstra, L., Poelman, H. \& Rodríguez-Pose, A. (2020) The geography of EU discontent. Regional Studies 54(6) 737-753. https://doi.org/10.1080/00343404.2019.1654603

ESPON (2011) Typology compilation. <https://www. espon.eu/tools-maps/regional-typologies> 15.5.2019.

Essletzbichler, J., Disslbacher, F. \& Moser, M. (2018) The victims of neoliberal globalisation and the rise of the populist vote: a comparative analysis of three recent electoral decisions. Cambridge Journal of Regions, Economy and Society 11(1) 73-94. https://doi.org/10.1093/cjres/rsx025
Faludi, A. (2006) From European spatial development to territorial cohesion policy. Regional Studies 40(6) 667-678. https://doi.org/10.1080/00343400600868937

Faludi, A. (2016a) The territoriality of cohesion policy. I: Piattoni, S. \& Polverani, L. (eds.) Handbook on cohesion policy in the EU. Edward Elgar, London.

Faludi, A. (2016b) EU territorial cohesion, a contradiction in terms. Planning Theory \& Practice 17(2) 302-313. https://doi.org/10.1080/14649357.2016.1154657.

Faludi, A., Stead, D. \& Humer, A. (2015) Services of General Interest, Territorial Cohesion and Competitiveness in Europe. I: Fassmann, H., Rauhut, D., Marques da Costa, E. \& Humer, A. (eds.) Services of General Interest and Territorial Cohesion: European Perspectives and National Insights. V\&R Univie Press, Göttingen.

Fratesi, U. \& Rodríguez-Pose, A. (2016) The crisis and regional employment in Europe: what role for sheltered economies? Cambridge Journal of Regions, Economy and Society 9(1) 33-57. https://doi.org/10.1093/cjres/rsv032

Gagliardi, L. \& Perocco, M. (2017) The impact of European Cohesion Policy in urban and rural regions. Regional Studies 51(6) 857-868. https://doi.org/10.1080/00343404.2016.1179384

Gruber, E., Rauhut, D. \& Humer, A. (2018) Territorial Cohesion under pressure? Welfare policy and planning responses in Austrian and Swedish peripheries. Papers in Regional Science 98(1) 115-132. https://doi.org/10.1111/pirs. 12344

Hadjimichalis, C. (2011) Uneven geographical development and socio-spatial justice and solidarity: European regions after the 2009 financial crisis. European Urban and Regional Studies 18(3) 254-274. https://doi.org/10.1177/0969776411404873

Hamez, G. (2005) Territorial Cohesion: How to Operationalize and Measure the Concept? Planning Theory \& Practice 6(3) 400-402. https://doi.org/10.1080/14649350500209454

Hanell, T. (2015) Measuring territorial cohesion: A macro regional approach. In: Lang, T., Henn, S., Sgibnev, W. \& Ehrlich, K. (eds.) Understanding geographies of polarization and peripheralization. Perspectives from Central and Eastern Europe and beyond. Palgrave Macmillan, London.

Isola, F., Leone, F. \& Pira, C. (2017) Towards a regional urban agenda: approaches and tools, Regional Studies, Regional Science 4(1) 181-188. https://doi.org/10.1080/21681376.2017.1356238

Lobao, L., Gray, M., Cox, K. \& Kitson, M. (2018) The shrinking state? Understanding the assault on the public sector. Cambridge Journal of Regions, Economy and Society 11(3) 389-408. https://doi.org/10.1093/cjres/rsy026 
Marques da Costa, E., Palma, P., Rauhut, D., Humer, A., Constantin, D. \& Velasco, X. (2013) What indicators to use when measuring services of general interests? Europa XXI 23 78-28. https://doi.org/10.7163/Eu21.2013.23.1

Medeiros, E. (2013) Assessing territorial impacts of the EU Cohesion Policy: the Portuguese case. European Planning Studies 22 (9) 1960-1988. https://doi.org/10.1080/09654313.2013.813910

Medeiros, E. \& Rauhut, D. (2020) Territorial Cohesion Cities: a policy recipe for achieving Territorial Cohesion? Regional Studies 54(1) 120-128. https://doi.org/10.1080/00343404.2018.1548764

Medve-Bálint, G. (2016) Funds for the wealthy and the politically loyal? How EU Funds may contribute to increasing regional disparities in East Central Europe. I: Bachtler, J., Berkowitz, P., Hardy, S. \& Muravska, T. (eds.) EU Cohesion Policy: Reassessing performance and direction. Routledge, London.

Meijers, E. \& Sandberg, K. (2008) Reducing Regional Disparities by Means of Polycentric Development: Panacea or Placebo? Scienze Regionali 7(2) 71-96.

Mendez, C. (2011) The Lisbonization of EU Cohesion Policy: A Successful Case of Experimentalist Governance? European Planning Studies 19(3) 519-537. https://doi.org/10.1080/09654313.2011.548368

Mohl, P. \& Hagen, T. (2010) Do EU structural funds promote regional growth? New evidence from various panel data approaches. Regional Science and Urban Economics 40(5) 353-365. https://doi.org/10.1016/j.regsciurbeco.2010.03.005

Molle, W. (2007) European cohesion policy. Routledge, London.

Nagy, J. \& Benedek, J. (2021) Does Cohesion Policy affect Territorial Inequalities and Regional Development? I: Rauhut, D., Sielker, F. \& Humer, A. (eds.) The EU's Cohesion Policy and Future Spatial Governance: Territorial, Economic and Social Challenges. Edward Elgar, Cheltenham. Under utgivning.

Partridge, M. D., Rickman, D. S., Rose Olfert, M. \& Tan, Y. (2015) When Spatial Equilibrium Fails: Is Place-Based Policy Second Best? Regional Studies 49(8) 1303-1325. https://doi.org/10.1080/00343404.2013.837999

Pellegrini, G., Terribile, F., Tarola, O., Muccigrosso, T. \& Busillo, F. (2012) Measuring the effects of European Regional Policy on economic growth: A regression discontinuity approach. Papers in Regional Science 92(1) 217-234. https://doi.org/10.1111/j.1435-5957.2012.00459.x

Rauhut, D. (2018) A Rawls-Sen Approach to Spatial Injustice. Social Science Spectrum 4(3) 109-122. $<$ http://www.socialspectrum.in/index.php/sp/article/ view/132/121> 26.8.2020.

Rauhut, D. \& Humer, A. (2020) The EU Cohesion Policy and Spatial Economic Growth: Trajectories in Economic Thought. European Planning Studies 28(11) 2116-2133. https://doi.org/10.1080/09654313.2019.1709416

Rodríguez-Pose, A. (2018) The revenge of the places that don't matter (and what to do about it). Cambridge Journal of Regions, Economy and Society 11(1) 189-209. https://doi.org/10.1093/cjres/rsx024

Servillo, L. (2019) Tailored polities in the shadow of the state's hierarchy. The CLLD implementation and a future research agenda. European Planning Studies 27(4) 678-698. https://doi.org/10.1080/09654313.2019.1569595

Solly, A. (2016) Place-based innovation in Cohesion Policy: meeting and measuring the challenges. Regional Studies, Regional Science 3(1) 193-198. https://doi.org/10.1080/21681376.2016.1150199

UNDP (2016a) Human Development Report 2016. United Nations, New York. <http://hdr.undp.org/sites/default/ files/HDR2016 EN Overview Web.pdf> 26.8.2020.

UNDP (2016b) Technical Notes - Calculating the human development indices. Unnited Nations, New York. <http://hdr.undp.org/sites/default/files/hdr2016 technical notes.pdf> 26.8.2020.

Ward, T. \& Wolleb, E. (2010) Ex-Post Evaluation of Cohesion Policy Programmes 2000-2006 financed by the European Regional Development Fund in Objective 1 and 2 Regions. CEC, Brussels.

World Bank (2018) World Bank database. <https://data. worldbank.org> 28.2.2018.

Zaucha, J. \& Böhme, K. (2020) Measuring territorial cohesion is not a mission impossible. European Planning Studies 28(3) 627-649. https://doi.org/10.1080/09654313.2019.1607827

Zaucha, J., Komornicki, T., Böhme, K., Swiatek, D. \& Zuber, P. (2014) Territorial Keys for Bringing Closer the Territorial Agenda of the EU and Europe 2020. European Planning Studies 22(2) 246-267. https://doi.org/10.1080/09654313.2012.722976 